Published in final edited form as:

Leuk Lymphoma. 2016 November ; 57(11): 2612-2618. doi:10.3109/10428194.2016.1166490.

\title{
Acute and late toxicity of bilateral orbital irradiation in the management of primary intraocular lymphoma
}

\author{
Sarah A. Milgrom ${ }^{a}$, Chan Y. Cheah ${ }^{b}$, Chelsea C. Pinnix ${ }^{a}$, Grace L. Smith ${ }^{a}$, Bouthaina S. \\ Dabaja $^{\mathrm{a}}$, Patricia Horace ${ }^{\mathrm{a}}$, Patricia Chevez-Barrios ${ }^{\mathrm{c}}$, Nathan H. Fowler ${ }^{\mathrm{b}}$, and Dan S. \\ Gombos $^{d}$ \\ aDepartment of Radiation Oncology, MD Anderson Cancer Center, Houston, TX, USA \\ ${ }^{b}$ Department of Medical Oncology, MD Anderson Cancer Center, Houston, TX, USA \\ 'Department of Pathology, MD Anderson Cancer Center, Houston, TX, USA \\ dDepartment of Ophthalmology, MD Anderson Cancer Center, Houston, TX, USA
}

\begin{abstract}
Primary intraocular lymphoma (PIOL) is a rare malignancy with poor outcomes. Concerns regarding toxicity lead some clinicians to exclude orbital radiation therapy (RT). We aimed to quantify the ocular toxicity of RT in 11 PIOL patients treated with chemoimmunotherapy and bilateral orbital RT (median 36 Gy). A multidisciplinary team, including an ocular oncologist, followed patients for a median of 42 months after RT. Common adverse events included dermatitis (100\%), conjunctivitis (82\%), xerophthalmia (64\%), and keratopathy (45\%). All phakic eyes developed cataracts $(100 \%)$; correction resulted in good vision recovery. New, visually significant retinopathy was observed in only one eye $(<5 \%)$ and affected a patient with preexisting diabetes. This report suggests that severe, vision-threatening complications following orbital RT are uncommon. In the absence of comorbidities, orbital RT should not be withheld due to fear of vision-threatening toxicity. The risk of toxicity may be augmented by comorbidities, so an individualized approach is recommended.
\end{abstract}

\section{Keywords}

Orbital; primary intraocular lymphoma; PIOL; radiation therapy; radiotherapy; RT

\section{Introduction}

\begin{abstract}
Primary intraocular lymphoma (PIOL), or vitreoretinal lymphoma, is an uncommon form of primary central nervous system lymphoma (PCNSL), in which disease involves the vitreous, retina, or optic nerve. As with other PCNSL, the most common histology is diffuse large Bcell lymphoma (DLBCL).[1] Because of its rarity, PIOL has been difficult to study, and the
\end{abstract}

Sarah Milgrom samilgrom@mdanderson.org Department of Radiation Oncology, Anderson Cancer Center, 1515 Holcombe Boulevard, Houston, TX, 77030, USA .

Potential conflict of interest: Disclosure forms provided by the authors are available with the full text of this article at http:// dx.doi.org/10.3109/10428194.2016.1166490. 
optimal treatment strategy remains to be defined. At our institution, we offer sequential RMPV (rituximab, methotrexate, procarbazine, and vincristine), radiation therapy (RT) targeting the bilateral orbits, and high-dose cytarabine. This management approach, extrapolated from the PCNSL experience,[2] aims to maximize local control and mitigate the risk of CNS relapse. Recently, we reported the clinical outcomes of this treatment strategy,[3] which compare favorably with results seen in other series. Notably, we observed a lower rate of intraocular disease relapse following combined modality therapy, when compared to strategies that omit RT.[4,5] Consistent with this finding, the International Extranodal Lymphoma Study Group reported on patients with intraocular lymphoma, with or without parenchymal brain involvement, who were treated with various approaches. Patients who received combined chemotherapy and RT experienced better ocular disease control and improved survival, compared to those treated with either modality alone.[6]

Despite these data, some members of the oncology community advocate withholding orbital RT out of concern for toxicity. A particularly feared complication is vision-threatening retinopathy.[7-14] No case of visually significant toxicity was identified in a recent report of 18 patients treated for PIOL with orbital RT. However, only six patients in this series received chemotherapy, in addition to RT.[15] It is possible that chemotherapy increases the incidence or severity of RT-related side effects. To investigate this concern, we assessed the acute and late ocular toxicity of RT in patients treated for PIOL using a uniform treatment strategy of chemoimmunotherapy and bilateral orbital RT.

\section{Materials and methods}

\section{Patients}

After obtaining a waiver of approval from our Institutional Review Board, we identified all patients treated for PIOL at our institution between 1 October 2007 and 1 April 2015. In each case, the diagnosis was established by pars plana vitrectomy, with specimens reviewed by an ocular cytopathologist. A complete staging evaluation consisted of a bilateral ophthalmologic examination, magnetic resonance imaging (MRI) of the brain, ${ }^{18} \mathrm{~F}$ fluorodeoxyglucose positron emission tomography-computed tomography (PET-CT) from the skull to mid-thighs, lumbar puncture with cerebrospinal fluid analysis, and bone marrow biopsy. The cohort for this study consisted of all patients with biopsy-proven PIOL and gross disease limited to the globes, who were treated with R-MPV, cytarabine, and RT targeting the bilateral orbits. Baseline characteristics, treatment details, and outcomes were retrieved from electronic medical records.

\section{Treatment}

Patients were offered treatment with R-MPV (rituximab $375 \mathrm{mg} / \mathrm{m}^{2}$ intravenously (IV) on day 1 , methotrexate $3.5 \mathrm{~g} / \mathrm{m}^{2}$ IV over two hours on day 1 followed by leucovorin rescue, procarbazine $100 \mathrm{mg} / \mathrm{m}^{2} /$ day orally for 7 days with odd cycles only, and vincristine 1.4 $\mathrm{mg} / \mathrm{m}^{2}$ capped at $2 \mathrm{mg}$ IV on day 1 ) given every two weeks for five cycles. RT was started a minimum of two weeks after the last cycle of methotrexate and targeted the bilateral orbits, including the entire globes. At least one month after the completion of RT, two cycles of rituximab (375 mg/m $\mathrm{m}^{2} \mathrm{IV}$ on day 1$)$ and cytarabine $\left(3 \mathrm{~g} / \mathrm{m}^{2} \mathrm{IV}\right.$ over three hours once daily 
on days $1+2$ ) were administered on two occasions, two weeks apart. Intrathecal (IT) methotrexate (12mg intrathecal or $6 \mathrm{mg}$ by Ommaya reservoir) or cytarabine (100 mg intrathecal) was typically administered once per cycle.

\section{Toxicity assessment}

Adverse events were recorded by treating clinicians at the time of occurrence, as part of standard management. During RT, patients were seen at least once weekly by the treating radiation oncologist and evaluated for side effects. After the completion of therapy, a multidisciplinary team, including an ocular oncologist (DSG), followed patients to assess for recurrence and treatment-related toxicity. Adverse events were graded using the Common Terminology Criteria for Adverse Events version 4.03.

\section{Results}

\section{Patient characteristics}

A total of 11 patients were treated for PIOL with R-MPV, bilateral orbital RT, and cytarabine. The median age was 66 years (range $48-72$ years), and nine patients (81\%) were female. All patients were negative for HIV infection at the time of diagnosis. The most common presenting symptoms ( $>1$ possible per patient) were floaters $(n=8,73 \%)$, blurred vision $(n=6,55 \%)$, and/or vision loss $(n=6,55 \%)$. Ophthalmologic examination revealed vitreous cells/opacity $(n=9,82 \%)$ and/or retinal/choroidal infiltrate $(n=4,36 \%)$. In all patients, gross disease was limited to the eyes; in one patient, cerebrospinal fluid (CSF) cytology was positive at diagnosis.

\section{Treatment characteristics}

Eight patients (73\%) completed all seven cycles of chemotherapy (5 R-MPV and 2 cytarabine); three had chemotherapy abbreviated due to toxicity and received 6, 5, and 4 cycles, respectively. All patients were treated with RT, targeting the bilateral orbits, to a median dose of $36 \mathrm{~Gy}$ in 20 fractions (range 30.6-39.6 Gy in 17-22 fractions). The technique for RT delivery was opposed tangential $6 \mathrm{MV}$ photon beams $(n=2 ; 18 \%)$, intensity modulated RT with $6 \mathrm{MV}$ photons $(n=3 ; 27 \%)$, or appositional $12-16 \mathrm{MeV}$ electron beams with custom skin collimation $(n=6 ; 55 \%)$. The dose and technique were selected based on the treating physician's preference. All patients completed the intended course of RT without interruption.

\section{Follow-up}

Patients were followed by a multidisciplinary team for a median of 42 months (range 8-94 months) after the completion of RT, with complete ophthalmologic examinations performed for a median of 35 months (range 6-72 months).

\section{Baseline ophthalmologic pathology}

Ocular pathology at baseline and during follow-up is summarized for each patient in Table 1. In this cohort, two patients had diabetes (18\%; cases 5 and 8), and one patient had unilateral diabetic retinopathy at the time of presentation (9\% of patients; $5 \%$ of eyes; case 5 ). Another 
patient (case 3) was severely visually impaired at diagnosis, due to neoplastic infiltrative retinopathy. Three patients were pseudophakic bilaterally at the time of diagnosis. Of the remaining eight patients, seven were noted to have bilateral grade 1 cataracts before initiating treatment for PIOL ( $88 \%$ of phakic eyes).

\section{Toxicity}

Several acute, self-limited complications were observed, none of which was significantly associated with RT technique. All patients developed radiation dermatitis of the periocular skin (7 grade 1, 4 grade 2). Dermatitis developed after patients had completed a median dose of 21.6 Gy (range 16.2-32.4 Gy) and resolved during the days to weeks following completion of RT (Figure 1). No chronic skin changes were observed. Nine patients (82\%) developed grade 1 conjunctivitis after receiving a median dose of 21.6 Gy (range 9-23.4 Gy), and five patients (45\%) developed keratopathy (2 grade 2, 3 grade 3 ) at a median of five weeks (range 3-12 weeks) after completion of RT. In all cases, conjunctivitis and keratopathy resolved either without intervention, or with conservative management, consisting of topical antibiotics, steroids, and/or lubricating ophthalmic ointments.

Xerophthalmia developed commonly in the acute period and persisted during long-term follow-up. Seven patients (64\%) developed symptomatic dry eye during or within two weeks after completion of RT. Five patients derived symptomatic relief from artificial tears and ointments (grade 1). Two patients required punctal occlusion (grade 2) at nine months and two years, respectively, after RT completion.

The most common late complication was cataract formation. Prior to RT, 14 of the 16 phakic eyes had grade 1 cataracts; during post-treatment ophthalmologic evaluations, all 16 phakic eyes developed grade 3 cataracts. In the single patient who did not have preexisting cataracts (case 2), bilateral cataracts were diagnosed 22 months after completion of RT. Vitrectomy, a risk factor for cataract formation [16], had been performed in 10 of the 16 phakic eyes (63\%). Surgical repair of cataracts was performed in 12 eyes, at a median of 27 months (range 3-43 months) after completion of RT, with good recovery of vision in all cases.

Retinopathy, a late complication of RT, was observed in two patients. Case 3 had diffuse retinal hemorrhage and proliferative change bilaterally, at the time of diagnosis, due to infiltration of the retina with lymphoma. Initially after therapy, this patient's visual impairment improved; however, 2.5 months later, she developed disease relapse in the bilateral vitreous. She experienced ongoing retinopathy and vision loss, due to progressive intraocular lymphoma (Figure 2). Among the eight patients whose disease did not relapse within the eye, there was one case of vision-threatening retinopathy. This patient (case 5) had long-standing type-2 diabetes mellitus and required concurrent treatment with pioglitazone, glyburide, and metformin to achieve glycemic control. She had grade 1 diabetic retinopathy of the right eye at diagnosis. After RT (total dose $37.8 \mathrm{~Gy}$, appositional electrons), she had persistent nonproliferative grade 1 retinopathy of the right eye. In the left eye, however, she developed sight-threatening, proliferative, grade 4 retinopathy, initially diagnosed 17 months after the completion of RT (Figure 3). She was treated with intravitreal bevacizumab, intravitreal steroids, and panretinal photocoagulation. Her vision declined from 20/40 at diagnosis to light perception at the time of last follow-up. 
Optic nerve damage, another severe complication, was observed in one patient. Case 3 had right optic nerve gliosis and pallor prior to initiating RT. As noted above, 2.5 months after completion of RT, her lymphoma relapsed in both eyes. She had progressive intraocular disease and visual decline. During follow-up, she was noted to have bilateral optic nerve pallor and sclerosis, with neoplastic infiltration. Conversely, in the eight patients who remained free of intraocular relapse, there was no case of optic neuropathy.

Visual acuity was recorded at the time of diagnosis and at the time of the last ophthalmologic examination. A measurement of 20/40 was used as a threshold to represent vision necessary for the activities of daily living and is commonly used to distinguish between grade 2 and grade 3 toxicity. At the time of diagnosis, visual acuity was 20/40 or better in 15/22 eyes (68\%). In the patients who did not experience an intraocular disease relapse, visual acuity at last follow-up was 20/40 or better in $9 / 16$ eyes (56\%); of note, poor vision at last follow-up was attributed to uncorrected cataracts in four eyes (cases 4,6 , and 11) and therefore was likely correctable. Of the eight patients who were free of intraocular disease, six (75\%) had visual acuity of 20/40 or better in at least one eye. Of the three patients who experienced an intraocular relapse, visual acuity was at least 20/40 in three of six eyes (50\%); two patients (67\%) had vision that was $20 / 40$ or better in one eye.

\section{Discussion}

Concern of site-threatening complications leads some clinicians to withhold orbital RT in the management of PIOL. In our series of 11 patients treated with R-MPV, bilateral orbital RT, and cytarabine, vision-threatening late effects were rare. Following RT, retinopathy was observed in only two cases. In one patient (case 3) secondary retinopathy was attributed to relapsed, refractory intraocular disease that infiltrated the retina. Additionally, this was the only case of optic neuropathy, in the setting of neoplastic infiltration of the nerve. Among the eight patients whose disease did not relapse in the eye, there was one case of severe retinopathy after RT. This patient (case 5) had longstanding type-2 diabetes and had grade 1 diabetic retinopathy at diagnosis. After RT (37.8 Gy), she developed significant, visionthreatening, proliferative retinopathy, which was refractory to multiple therapies and was attributed to the combination of her underlying vasculopathy (diabetes) and ocular RT.

Radiation retinopathy can be a severe complication of orbital RT. It may present with capillary occlusion and dilatation, microaneurysm and telangiectasia formation, neovascularization, and retinal pigment epithelial changes. It occurs most commonly between six months and three years after the completion of RT.[17] Its incidence depends on RT dose and fractionation. Parsons et al. reported on 27 eyes with visually significant radiation retinopathy. In that series, no patient had received below $45 \mathrm{~Gy}$ to the retina, suggesting that this dose may represent a threshold for clinically significant retinopathy. It is accepted that the risk of retinopathy is low when the retina is treated to less than $45 \mathrm{~Gy}$. All patients in the current series received less than this dose. However, the effect of RT may be augmented by comorbidities and chemotherapy administration, and there are reports of radiation retinopathy occurring at doses of 30-36 Gy administered in 2 Gy fractions.[11,14] Case 5 in this report had underlying diabetes and developed significant proliferative retinopathy after receiving $37.8 \mathrm{~Gy}$ in $1.8 \mathrm{~Gy}$ fractions. 
With the caveat of small sample size, our findings suggest that underlying vasculopathy, such as diabetes, may increase patients' susceptibility to progressive retinopathy after RT and may reduce the threshold dose for this toxicity. Congruent with this hypothesis, other researchers have identified diabetes as a significant risk factor for retinopathy in patients receiving RT for orbital lymphoma,[12] as well as choroidal melanoma $(p<0.001)$.[9] It has been hypothesized that there may be a synergism between diabetic and radiation retinopathy, because diabetic retinopathy results in loss of pericytes, whereas radiation retinopathy results in loss of endothelial cells. Pericytes and endothelial cells are the primary cell types of retinal capillaries, so the concomitant effect of diabetes and RT may leave little cellular support for capillaries, resulting in hemorrhage and exudation.[11]

While radiation retinopathy was rarely observed in this series, cataracts were a common late effect. Cataracts are a manageable complication, given the safe and effective procedures available for their correction. Ionizing radiation is a well-known risk factor for cataract formation. RT causes extensive cell death in the germinative zone of the lens epithelium, which, in turn, triggers compensatory cellular mitosis and differentiation. This wave of proliferation disrupts the precise organization of the fiber cells, resulting in cataractogenesis. [17] In this series, all phakic eyes had progressive cataracts during post-treatment follow-up. Of note, the majority had undergone vitrectomy, another precipitant for cataract formation.

[16] Other risk factors for cataract development in this population included steroid treatment, chronic uveitis, and advanced age. Cases that underwent subsequent cataract extraction maintained good visual acuity.

Vision preservation was a critical endpoint of our study. Visual acuity of 20/40 was used as a threshold to represent vision necessary for the activities of daily living and is commonly used to distinguish between grade 2 and grade 3 toxicity. At diagnosis, visual acuity was $20 / 40$ or better in $15 / 22$ eyes $(68 \%)$. In patients who did not relapse in the eye, visual acuity at last follow-up was at least 20/40 in 9/16 eyes (56\%); of note, in four eyes with visual acuity worse than $20 / 40$, poor vision was attributed to uncorrected cataracts and, therefore, we suspect was correctable. Conversely, in patients who experienced an intraocular relapse, visual acuity was $20 / 40$ or better in three of six eyes (50\%). We are not aware of any other reports of late visual acuity in patients treated for PIOL.

The most common adverse event, affecting all patients, was an acute, self-limited, grade 1-2 dermatitis of the periocular skin. The initial reaction occurred after approximately two weeks of fractionated RT, correlating with the time required for cell migration from the basal to the keratinized layer of the skin.[17] In all cases, dermatitis resolved during the days to weeks following completion of RT. There was no case of chronic skin change in the irradiated area.

The other acute effects of RT included xerophthalmia, conjunctivitis, and keratopathy. Xerophthalmia may result from damage to glands within the eyelid, reduction in conjunctival mucus production, and decrease in lacrimal gland secretion.[17] In our series, xerophthalmia was common (64\% of patients). In most cases, lubricants were sufficient for symptomatic relief. Consistent with our finding, Kennerdell et al. reported that mild xerophthalmia affected $50 \%$ of patients acutely and $33 \%$ of patients chronically, after 
treatment for low-grade orbital lymphoma with RT to a total dose of 24-25.5 Gy.[18] The greater incidence in our cohort is likely due to the higher RT dose and administration of chemotherapy. Tear film dysfunction may contribute to temporary conjunctivitis or keratopathy after RT.[17] In this series, conjunctivitis and keratopathy were observed in $82 \%$ and $45 \%$ of patients, respectively. Topical steroids, antibiotics, and lubricating ointments alleviated discomfort, and all cases resolved within a few weeks to months of RT.

Taken together, our findings suggest that severe complications of orbital RT in the management of PIOL are rare. Visually significant proliferative retinopathy occurred in only one eye $(<5 \%)$ and affected a patient with comorbid diabetes and preexisting retinopathy. These findings are supported by a recent report of 18 patients treated with orbital RT for PIOL, none of whom developed vision-threatening toxicity.[15] We conclude that oncologists should not withhold orbital RT due to fear of sight-threatening retinopathy, in the absence of comorbidities. However, they should use caution when treating patients with comorbid conditions, such as diabetes. If RT is deemed necessary, diabetic patients should undergo careful retinal examinations at baseline and regularly during follow-up after RT.

To the best of our knowledge, this is the first report to focus on the toxicity of orbital RT in patients treated for PIOL. A major strength of our paper is the long follow-up, with routine ophthalmologic evaluations by an experienced ocular oncologist (DSG). Another strength is the uniform treatment approach that was used for all patients. Limitations include its retrospective nature and small sample size.

PIOL is an uncommon form of lymphoma, with a poor prognosis. Local recurrence remains a major hurdle to preservation of vision and quality of life, even in patients treated with intensive chemo-immunotherapy and RT targeting the bilateral orbits.[3] New therapeutic approaches, such as high-dose chemotherapy with autologous stem cell rescue, are an area of active study. At this time, RT, which aims to maximize local disease control, maintains an important role in the armamentarium against PIOL. Vision-threatening toxicity of RT is rare. The risk is likely higher in patients with underlying vasculopathy, so an individualized approach is critical. Further study will shed greater insight into approaches to optimize disease control, while minimizing the morbidity of therapy.

\section{References}

[1]. Chan CC, Rubenstein JL, Coupland SE. Primary vitreoretinal lymphoma: a report from an international primary central nervous system lymphoma collaborative group symposium. Oncologist. 2011; 16:1589-1599. [PubMed: 22045784]

[2]. Morris PG, Correa DD, Yahalom J. Rituximab, methotrexate, procarbazine, and vincristine followed by consolidation reduced-dose whole-brain radiotherapy and cytarabine in newly diagnosed primary CNS lymphoma: final results and long-term outcome. J Clin Oncol. 2013; 31:3971-3979. [PubMed: 24101038]

[3]. Cheah CY, Milgrom S, Chihara D. Intensive chemoimmunotherapy and bilateral globe irradiation as initial therapy for primary intraocular lymphoma. Neuro Oncol. 2016; 18:575-581. [PubMed: 26487691]

[4]. Hormigo A, Abrey L, Heinemann MH. Ocular presentation of primary central nervous system lymphoma: diagnosis and treatment. Br J Haematol. 2004; 126:202-208. [PubMed: 15238140]

[5]. Soussain C, Merle-Beral H, Reux I. A single-center study of 11 patients with intraocular lymphoma treated with conventional chemotherapy followed by high-dose chemotherapy and 
autologous bone marrow transplantation in 5 cases. Leuk Lymphoma. 1996; 23:339-345. [PubMed: 9031115]

[6]. Ferreri AJ, Blay JY, Reni M. Relevance of intraocular involvement in the management of primary central nervous system lymphomas. Ann Oncol. 2002; 13:531-538. [PubMed: 12056702]

[7]. Robertson DM, Buettner H, Gorman CA. Retinal microvascular abnormalities in patients treated with external radiation for graves ophthalmopathy. 2003. Arch Ophthalmol. 121:652-657.

[8]. Trikha R, Morse LS, Zawadzki RJ. Ten-year follow-up of eyes treated with stereotactic fractionated external beam radiation for neovascular age-related macular degeneration. Retina. 2011; 31:1303-1315. [PubMed: 21499195]

[9]. Gragoudas ES, Li W, Lane AM. Risk factors for radiation maculopathy and papillopathy after intraocular irradiation. Ophthalmology. 1999; 106:1571-1577. [PubMed: 10442906]

[10]. Kumar B, Palimar P. Accelerated radiation retinopathy in diabetes and pregnancy. Eye (Lond). 2000; 14:107-108. [PubMed: 10755117]

[11]. Viebahn M, Barricks ME, Osterloh MD. Synergism between diabetic and radiation retinopathy: case report and review. Br J Ophthalmol. 1991; 75:629-632. [PubMed: 1954216]

[12]. Kaushik M, Pulido JS, Schild SE. Risk of radiation retinopathy in patients with orbital and ocular lymphoma. Int J Radiat Oncol Biol Phys. 2012; 84:1145-1150. [PubMed: 22592046]

[13]. Hoffman PM, McKelvie P, Hall AJ. Intraocular lymphoma: a series of 14 patients with clinicopathological features and treatment outcomes. Eye (Lond). 2003; 17:513-521. [PubMed: 12802353]

[14]. Hata M, Kaneko A, Tomita N. Severe retinopathy following radiation therapy with a moderate dose for orbital mucosa-associated lymphoid tissue lymphoma. Hematol Oncol. 2014; 32:212214. [PubMed: 25471380]

[15]. Teckie S, Yahalom J. Primary intraocular lymphoma: treatment outcomes with ocular radiation therapy alone. Leuk Lymphoma. 2014; 55:795-801. [PubMed: 23822539]

[16]. de Bustros S, Thompson JT, Michels RG. Nuclear sclerosis after vitrectomy for idiopathic epiretinal membranes. Am J Ophthalmol. 1988; 105:160-164. [PubMed: 3341433]

[17]. Durkin SR, Roos D, Higgs B. Ophthalmic and adnexal complications of radiotherapy. Acta Ophthalmol Scand. 2007; 85:240-250. [PubMed: 17488452]

[18]. Kennerdell JS, Flores NE, Hartsock RJ. Low-dose radiotherapy for lymphoid lesions of the orbit and ocular adnexa. Ophthal Plast Reconstr Surg. 1999; 15:129-133. 


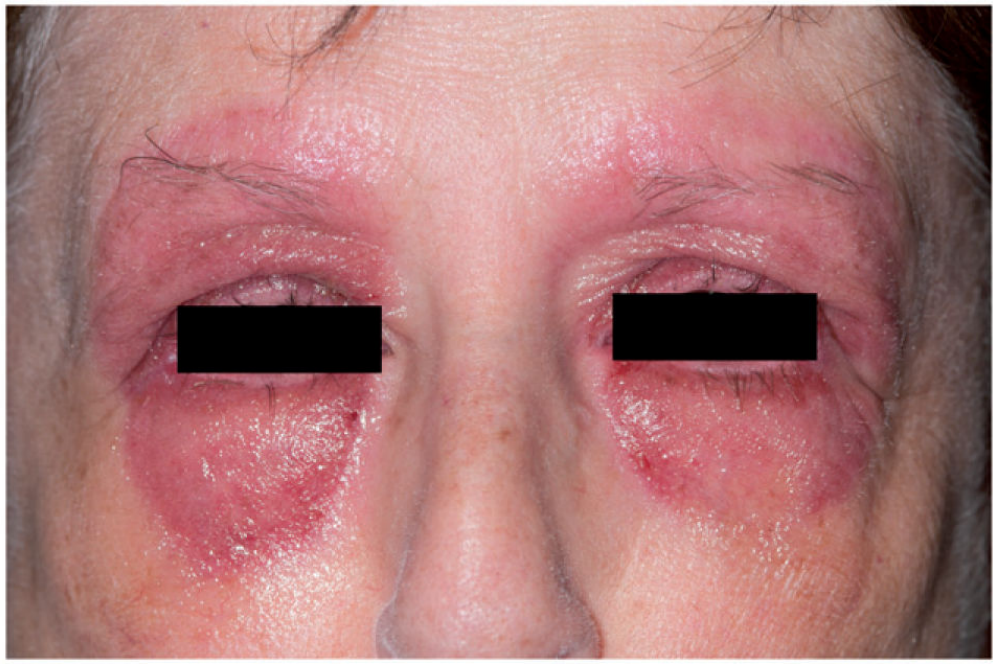

Figure 1.

Grade 2 dermatitis at the completion of radiation therapy to a total dose of 32.4 Gy in 18 fractions, using $12 \mathrm{MeV}$ appositional electron fields and custom skin collimation. 


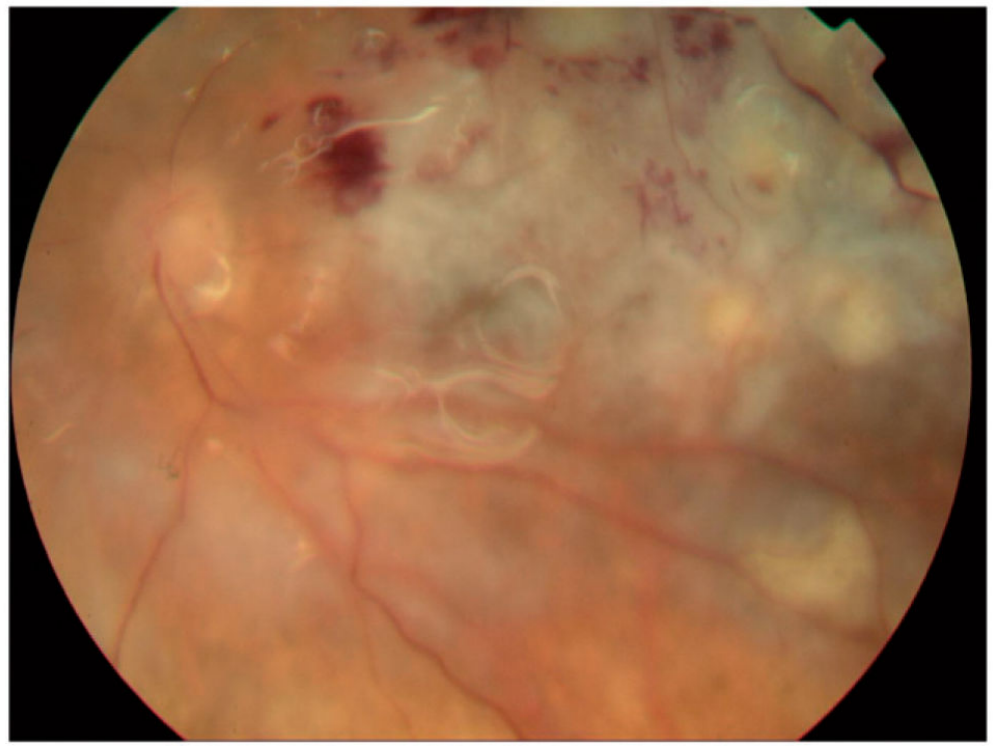

Figure 2.

Neoplastic infiltrative retinopathy and optic neuropathy. 

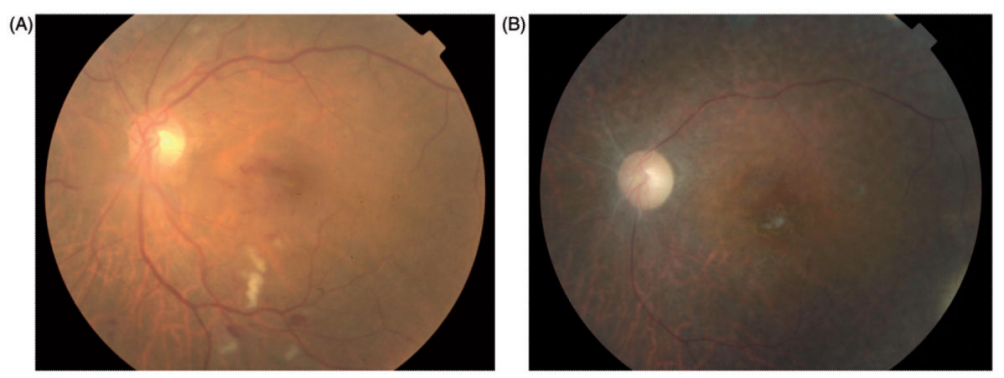

Figure 3.

Grade 4 retinopathy following orbital radiation therapy $(37.8 \mathrm{~Gy})$ in a patient with comorbid diabetes. (A). Retinal changes following treatment with intravitreal bevacizumab, intravitreal steroids, and panretinal photocoagulation (B). 
Milgrom et al.

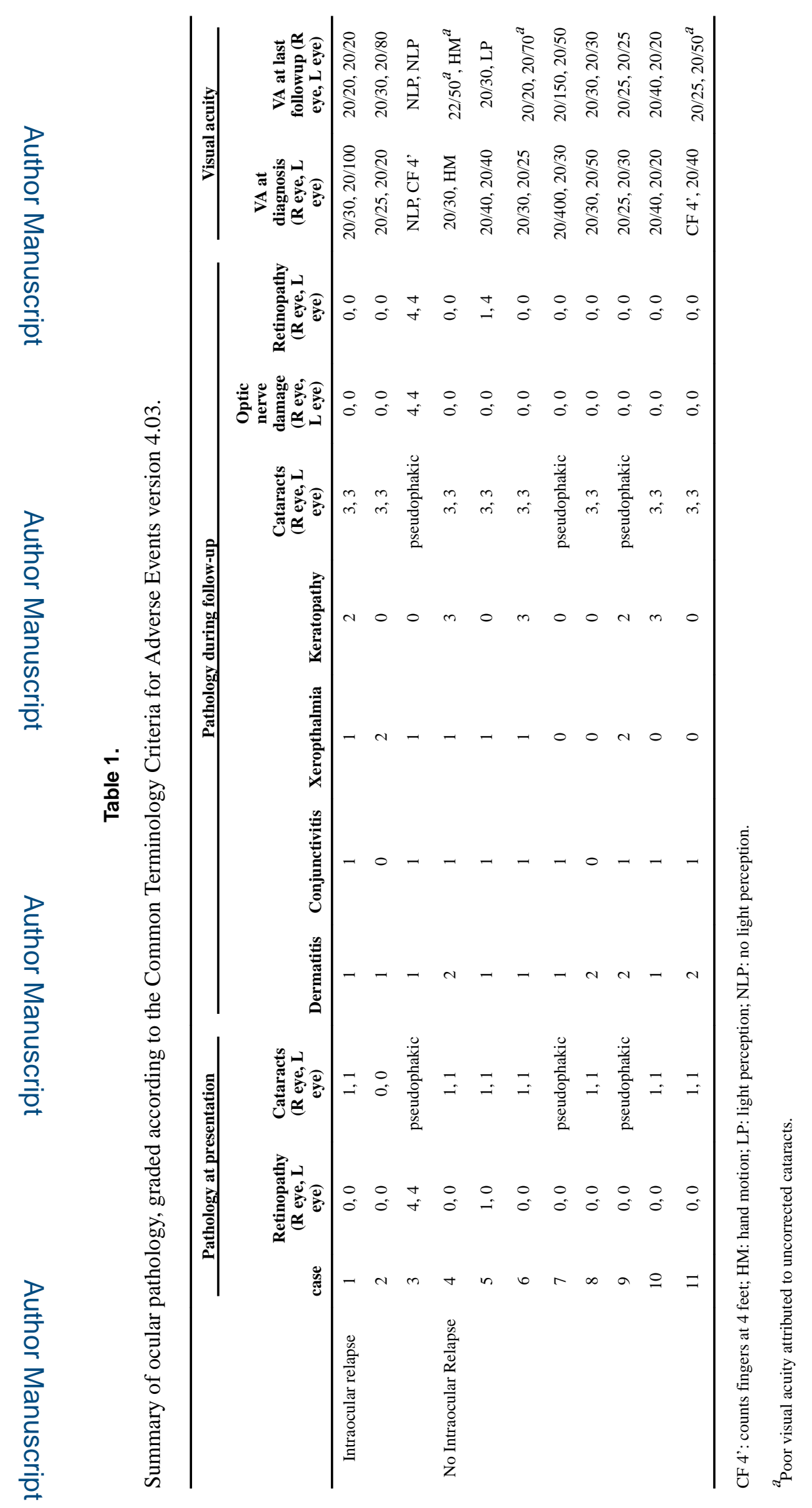

Leuk Lymphoma. Author manuscript; available in PMC 2018 July 16. 